\title{
Biología y calidad del agua del acuífero norte \\ de Quintana Roo \\ DOI: 10.22403/UQROOMX/TYP03/11
}

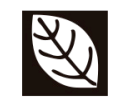

\section{RESUMEN}

Martha Gutiérrez Aguirre *

En esta nota de investigación se revisa el estado de conocimiento del acuífero norte de Quintana Roo, particularmente los estudios referentes al conocimiento biológico y a la calidad y cantidad de agua albergada en las corrientes subterráneas de la región. La zona norte de Quintana Roo presenta una alta densidad turística con una gran demanda del recurso agua. Las evidencias muestran que de manera general poco se sabe de la calidad, cantidad y flujos de las corrientes subterráneas de la región; en el corto plazo será necesario que se fomente su estudio, lo cual ayudaría a identificar áreas de protección y de contaminación de corrientes subterráneas.

Palabras $\mid$ Acuífero, cárstico, evaluación, Quintana Roo, subterráneo.

*Universidad de Quintana Roo, Cuerpo Académico de Turismo / margutierrez@uqroo.mx 


\section{Introducción}

En regiones donde dominan suelos cársticos los flujos de agua son principalmente subterráneos (Alcocer y Escobar, 1996: 55; Herrera y Comín, 2000:2 1 3; Schmitter et al., 2002:217). Éstos transportan recursos acuáticos para el uso y consumo humano, y soportan gran cantidad de biomasa terrestre.

En la región sureste de México se capta alrededor de $50 \%$ de la precipitación anual del país; se constituye como la zona de captación de agua dulce de mayor importancia, considerando que México únicamente tiene acceso a $0.1 \%$ de las reservas del vital líquido a nivel mundial (Alcocer y Escobar, 1996:57).

Dentro de la región sureste del país, particularmente en la Península de Yucatán es característica la presencia de un suelo calcáreo que, entre otras cosas, se distingue por sufrir un proceso altamente dinámico conocido como carstificación, el cual consiste en la disolución de la roca (compuesta de $\mathrm{CaCO}_{3}$ ), en la presencia de ácido carbónico $\left(\mathrm{HCO}_{3}-\right)$; producto de la reacción entre el bióxido de Carbono $\left(\mathrm{CO}_{2}\right)$ y el agua $\left(\mathrm{H}_{2} \mathrm{O}\right)$ (Suárez y Rivera, 2000: I5I). Esta sencilla relación produce los sistemas acuáticos típicos de la región (cenotes), con dominio de corrientes subterráneas.

Debido a que estos sistemas acuáticos constituyen la principal fuente de agua dulce para la población y soportan una gran biodiversidad, en la presente nota se realiza una revisión del estado de conocimiento de los sistemas acuáticos de la Península de Yucatán, particularmente del acuífero norte de Quintana Roo, poniendo particular énfasis en los estudios realizados a nivel biológico y de calidad del agua.

\section{Conocimiento biológico y de calidad del agua}

Los estudios realizados en sistemas acuáticos cársticos de la Península de Yucatán se han enfocado particularmente en dos aspectos: I) geohidrológicos, geoquímicos y de contaminación (Marín y Perry, 1994:623; Steinich et al., I996a: I 59;Alcocer et al., 1998: 30 I; Herrera y Comín, 2000: 227; Socki et al., 2002: I818); y 2) taxonómicos (Suárez et al., 1996: 296; Schmitter, 1998: 239; Suárez y Reid, 1998: 265; Alcocer et al., 1999: 25; Sarma y Elías, 1999: 196; Elías y Suárez, 2000: I4).

Aunque los trabajos antes citados representan grandes esfuerzos de investigación, es notable que apenas durante la última década los estudios a nivel geomorfológico e hidrológico hayan adquirido mayor importancia. 
Considerando la extensión que ocupan los terrenos cársticos en la Península de Yucatán, es claro que sólo pequeñas regiones geográficas han sido estudiadas, tal es el caso de la región noroeste de Yucatán conocida como la zona "altamente variable" que ha sido profundamente examinada (Steinich et al., 1996: I59; Steinich y Marín, 1997: 331).

Debido a lo anterior, en amplias regiones de la Península (incluida la región norte de Quintana Roo) se desconoce la dirección, escorrentía, calidad, volumen y velocidad de los flujos de agua subterránea; así como los puntos de afloramiento en áreas costeras del agua procedente de áreas continentales (Steinich y Marín, 1997: 320; Schmitter et al., 2002: 220).

A nivel biológico, la situación es semejante: el conocimiento sobre los organismos vertebrados e invertebrados que habitan este tipo de sistemas es escaso; aún no existen referencias publicadas en relación con los protozoarios, hidrozoarios, nemátodos, anélidos o moluscos de estos sistemas. La mayoría de los estudios se enfocan en macrocrustáceos y zooplancton (Suárez y Rivera, 2000: 160) y los mayores esfuerzos de muestreo se han concentrado en la región centro-este de Quintana Roo, dejando a la región norte del estado, nuevamente, con escaso conocimiento de la diversidad que habita sus sistemas acuáticos más característicos.

La isla de Cozumel es uno de los polos turísticos más importantes del país; ocupa el primer lugar mundial como destino de cruceros (Lozano, 2002: 5I), y su número de habitantes, así como el consumo de agua, se han incrementado exponencialmente en los últimos 30 años (Wurl y Giese, 2005: 173). Sobre esta isla de origen cárstico, al igual que de algunas regiones de la Península, hasta ahora se sabe que el acuífero está constituido por una delgada capa de agua dulce que flota sobre una de agua salina más densa (Doehring y Butler, 1974: 592; Lesser y Weidie, 1998). Sin embargo, no son conocidas las características básicas del acuífero a nivel geohidrológico ni biológico, cuyo conocimiento es útil para detectar flujos subterráneos, los cuales subsecuentemente proporcionarían información para: I) observar posibles impactos y condiciones de calidad de agua en el acuífero (e incluso en el ambiente costero asociado a él); y 2) hacer evaluaciones del balance hídrico a nivel regional, que actualmente son incipientes en la región.

Debido al escaso conocimiento, no es posible señalar con certeza las áreas con influencia antrópica o la magnitud de los contaminantes sobre el acuífero de la región norte de Quintana Roo; a su vez, también se desconoce cómo 
impactan a la franja costera inmediata del litoral (la cual soporta a la comunidad del arrecife), tampoco se ha establecido la posible variación espacial y temporal de las concentraciones de nutrientes o contaminantes arrastrados desde el área continental $y$, además, se desconocen los puntos de afloramiento de agua dulce en el ambiente costero en las áreas de influencia urbana.

Se hace necesario detectar los anteriores factores para realizar propuestas para el mejor uso y aprovechamiento del agua, debido a que en las últimas décadas, a través de indicadores de productividad del agua, se ha observado una tendencia hacia la mesotrofia, es decir, la apariencia más o menos normal de estar bien nutrido pero que en realidad tiene un nivel degradado de salud (lo anterior se ha reportado en sistemas del norte de Yucatán) en cuerpos de agua que anteriormente se consideraban oligotróficos y que ahora son influenciados por actividades de producción ganadera o por la cercanía de grandes ciudades (véase cuadro I).

\begin{tabular}{lc}
\hline Indicador $(\mathrm{mg} / \mathrm{l})$ & Concentración \\
\hline Fosfato & ND -0.7 \\
Nitrato & $0.05-3.0$ \\
Nitrito & ND -15 \\
Clorofila $\alpha$ & $0.11-97.4$ \\
\hline
\end{tabular}

Fuente: Alcocer et al., 1998: 295 y Flores Nava, 1994: 90.

Cuadro I. Rangos en los valores de Indicadores de productividad DEL AGUA

\section{Conclusiones}

El escaso conocimiento de las características básicas del agua, así como de los organismos que la habitan, limita el uso correcto y el aprovechamiento de los sistemas acuáticos subterráneos y superficiales de la región norte de Quintana Roo.A pesar de que en las últimas décadas se ha incrementado el interés por entender el comportamiento básico de las corrientes subterráneas mediante estudios geohidrológicos y de comportamiento físico y químico del agua, a nivel regional aún quedan grandes áreas prácticamente inexploradas y son precisamente las que actualmente o a corto plazo tendrán mayor importancia como regiones de gran demanda de agua debido al turismo. 
Algunas herramientas que podrían emplearse para describir las características básicas de los sistemas cársticos son la identificación de parámetros ambientales considerados como elementos traza (ET) reactivos (por ejemplo el $\mathrm{NO}_{3}, \mathrm{NO}_{2}, \mathrm{Na}-, \mathrm{K}+, \mathrm{SO}_{4}, \mathrm{Cl}-$ ) y no reactivos ( $\mathrm{pH}$, la conductividad, salinidad, etcétera). Mediante estos parámetros se puede detectar el flujo subterráneo local, a partir de las concentraciones de los elementos traza tanto en los sistemas cársticos continentales como en la franja costera.

Por otra parte, con el estudio de variables físicas y químicas (salinidad, nutrimentos, etc.) se puede conocer la calidad del agua que guardan los sistemas acuáticos superficiales y subterráneos, así como el aporte de los cuerpos de agua superficiales continentales hacia los costeros.

Una herramienta adicional que proporcionaría información acerca de los flujos de agua subterránea es el Sistema de Información Geográfico (SIG); donde el uso de mapas temáticos ayudaría a clasificar los diferentes tipos de afloramientos de agua de la región. Esta información, junto con bases de datos de características físicas y químicas del agua permitiría, por un lado, describir el comportamiento básico de los sistemas acuáticos más característicos de la Península de Yucatán; por el otro, sería posible identificar la dispersión de contaminantes a través de los flujos subterráneos, las fuentes potenciales para uso de agua potable y áreas de protección de recursos acuáticos subterráneos.

\section{Agradecimientos}

El presente trabajo es resultado de la colaboración entre los cuerpos académicos de Turismo y Geohidrología. La colaboración se facilitó a través del apoyo de Rafael González Plascencia, Oscar Frausto Martínez, Adrián Cervantes Martínez, y los miembros del Cuerpo Académico de Turismo (CAT), por la Universidad de Quintana Roo, Unidad Académica Cozumel; así como por Lars Matthes y Uwe Troeger por la Universidad Técnica de Berlín.

\section{FUENTES CONSULTADAS}

Alcocer,J.\& E. Escobar ( 1996). "Limnological regionalization of Mexico”. Lakes and Reservoirs: Research and Management, 2, 55-69.

Alcocer,J.,A. Lugo., L. E. Marín \& E. Escobar (1998)."Hydrochemistry of waters from five cenotes and evaluation of their suitability for drinking-watersupplies, northeastern Yucatan, Mexico”. Hydrgeology Journal, 6, 293-30I. 
Alcocer A., A. Lugo., M. R Sanchéz., E. Escobar \& M. Sánchez (1999). "Bacterioplankton from cenotes and anchialine caves of Quintana Roo, Yucatan Peninsula, México". Revista de Biología Tropical, 47, 19-25

Doehring, D. O. \& J. H. Butler (1974). "Hydrogeologic constrains on Yucatan's development”. Science, I86, 59I-595.

Elías-Gutiérrez M. \& E. Suárez-Morales (2000)."Estudios sobre el zooplancton epicontinental en México: historia, estado actual y nuevos hallazgos”. Mexico, I, 9-I4.

Flores-Nava, A. (1994). "Some limnological data from five water bodies of Yucatán as a basis for aquaculture development". Anales del Instituto de Ciencias del Mar y Limnología, 21, 87-98.

Herrera-Silveira,J.A.\& F.A. Comín (2000). "An introductory account of types of aquatic ecosystems of Yucatan Peninsula (SE Mexico)", en Munawar M., S. G. Lawrence, I. F. \& D. F. Malley (eds.). Aquatic ecosystems of Mexico. Status and Scope. The Netherlands: Backhuys Publishers, 2 I 3-227.

Lesser, J.A. \& A. E.Weidie. (1998). “Region 25, Yucatan Peninsula”, en Back,W., Rosenshein,J.S.\& Seaber, P. R. (eds.). Hydrogeology:Boudler, Colorado, Geological Society of America, The Geology of North America (v. O-2). USA: Geological Society of America.

Lozano, M. (2002). "Turismo seguro en la isla de Cozumel”, en O. FraustoMartínez (ed.). Memorias del 3er Taller Internacional sobre turismo en el Caribe. México: Universidad de Quintana Roo, 5I-56.

Marín L.E.\& E. C.Perry.(1994).“The hydrogeology and contamination potential of north western Yucatan, Mexico”. Geofisica Internacional, 33, 619-623.

Sarma S.S.S. \& M. Elías-Gutiérrez. (1999). “A survey on the rotifer (Rotifera) fauna of the Yucatan Peninsula (Mexico)”. Revista de Biología Tropical, 47, 187-196.

Schmitter-Soto J. J. (1998). Catálogo de los peces continentales de Quintana Roo. México: El Colegio de la Frontera Sur.

, F.A. Comín, E. Escobar-Briones, J.Herrera-Silveira,J.Alcocer, E. SuárezMorales, M. Elías-Gutiérrez, V. Díaz-Arce, L. E. Marín \& B. Steinich. (2002)."Hydrogeochemical and Biological characteristics of cenotes in the Yucatan Peninsula (SE Mexico)". Hydrobiologia, 467, 21 5-228.

Socki, R.A. E. C. Perry, Jr. \& C. S. Romanek (2002). "Stable isotope systematics of two cenotes from the northern Yucatan Peninsula, Mexico". Limnol. Oceanogr, 47, I808-1818. 
Steinich B. \& L. E. Marín (1997. "Determination of flow characteristics in the aquifer of the Northwestern Peninsula of Yucatan, Mexico". Journal of Hydrobiology, I9I, 315-331.

Steinich B., G.V. Olimán., L. E. Marín \& E. Perry (1996). “Determination of the ground water divide in the karst aquifer of Yucatan, Mexico, combining geochemical and hydrogelogical data". Geofisica Internacional, 35, I53-159.

Suárez- Morales E. \& J.W. Reid (1998). "An updated list of the free-living freshwater copepods (Crustacea) of Mexico". The Southwestern Naturalist, 43, 256-265.

Suárez-Morales, E.,J.W. Reid,T. M. lliffe \& F. Fiers (1996). Catálogo de los copépodos (Crustacea) continentales de la Península de Yucatán, México. México: Comisión Nacional para el Conocimiento y Uso de la Biodiversidad y El Colegio de la Frontera Sur.

Suárez-Morales E. \& E. Rivera-Arriaga (2000). "The aquatic fauna of karstic environments in the Yucatan Peninsula, Mexico:an updated overview", en Munawar M., S. G. Lawrence, I. F. Munawar \& D. F. Malley (eds.). Aquatic ecosystems of Mexico. Status \& Scope. The Netherlands: Backhuys Publishers, I5I-I64.

Wurl, J. \& S. Giese (2005). Ground water quality research on Cozumel island, State of Quintana Roo, Mexico, en O. Frausto-Martínez (ed.). Desarrollo Sustentable:turismo, costas y educación. México: Universidad de Quintana Roo, I7I-I76. 


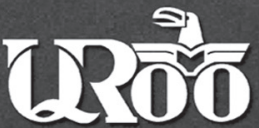

\section{UNIVERSIDAD DE OUINIANA ROO}

UNIDAD COZUMEL

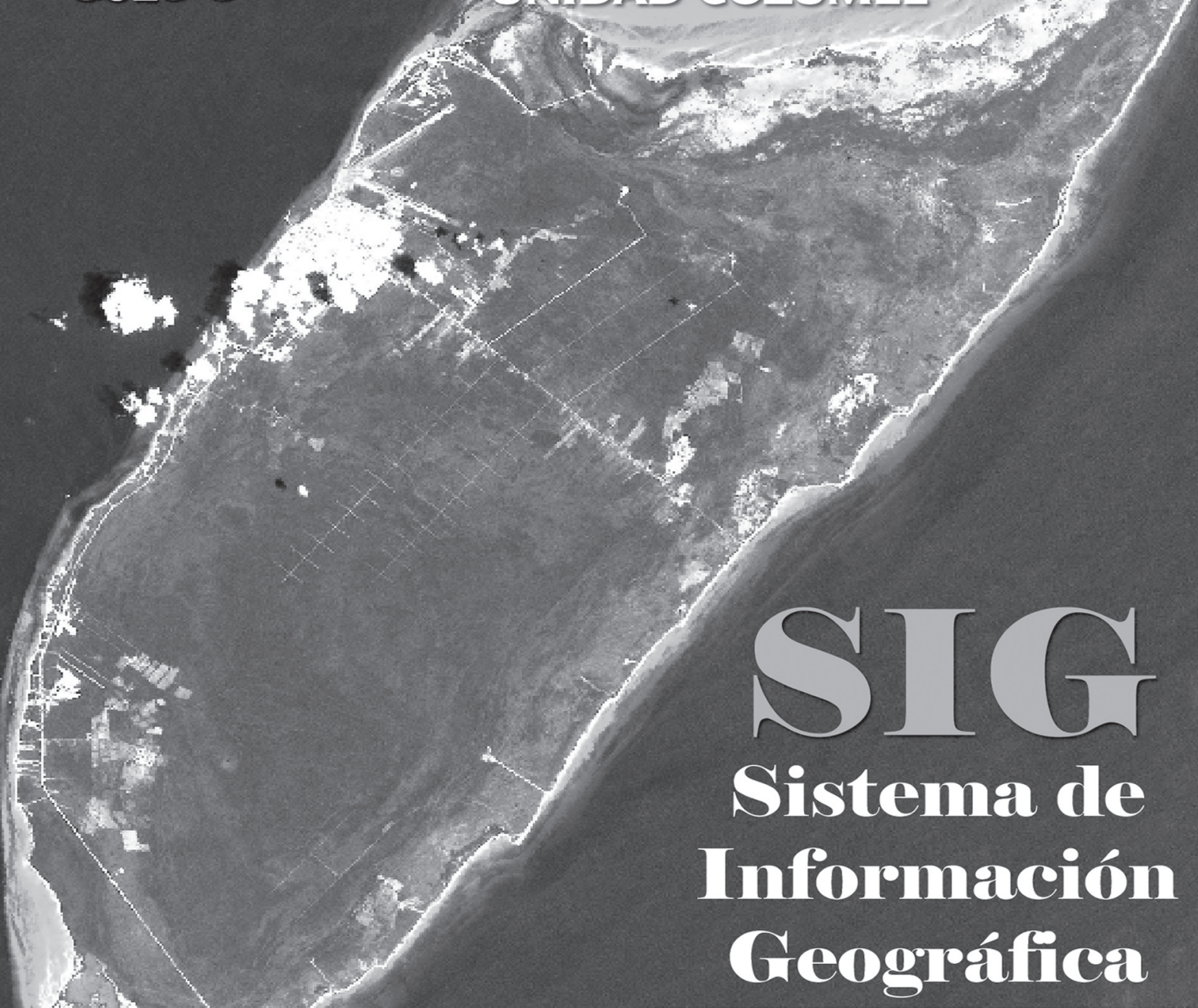

El Sistema de Información Geográfica de la Universidad de Quintana Roo, con apenas tres años de servicios, ha tenido importantes logros en la adquisición y creación de software y hardware.

Entre las ventajas que podría tener este Sistema de Información Geográfica para una comuna como la de Cozumel figura el manejo del Catastro, de la información sobre negocios, de Protección civil, de Desarrollo Urbano, de Protección al Medio ambiente, entre otros grandes rubros. 